\title{
Agricultural Economics Case Teaching and Effectiveness Evaluation based on Markov Chain
}

\author{
Shuang Yu, Guang $\mathrm{Li}^{*}$ and Yulian Ye
}

College of Resource and Environment, Anshun University, Anshun 561000

*the corresponding author

Keywords: Case teaching; Agricultural Economics; Markov chain; Teaching evaluation

Abstract. Teaching evaluation is a basic and indispensable link in the teaching activities. It has become an important urgent task of all kinds of school to establish and improve the teaching evaluation system. In this paper, Limit distribution and Penalty factor method of Homogeneous Markov Chain are evaluation of teaching effectiveness about Case teaching mode in "Agricultural Economics" course teaching process, evaluation results are objective and accurate. Results showed that the implementation of case teaching students good teaching effect in the implementation of case teaching are not students, illustrate the case teaching in the teaching of mathematics is an effective teaching method.

\section{基于马尔科夫链的《农业经济学》案例式教学模式 的应用效果评价}

\author{
余霜，李光，叶玉莲 \\ 安顺学院资源与环境工程学院, 安顺 561000
}

摘要: 教学评价是教学活动中不可缺少的一个基本环节, 建立和完善教学评价体系, 已成为各类学校急需解决的重要任 务。本文利用齐次马尔科夫链的极限分布和罚因子法对案例式教学模式在《农业经济学》课程教学过程中的教学效果进行评 价, 评价结果客观准确。结果表明: 案例式教学模式教学效果好于常规教学模式, 说明案例式教学模式在《农业经济学》教 学中是一种行之有效的教学方法。

关键词: 案例式教学模式; 农业经济学; 马尔科夫链; 教学评价

\section{1 前言}

案例教学起源于 “哈佛大学” 的情景案例教学课, 历经百年发展, 案例式教学现已成为现代教育教学 的重要方法之一。案例式教学模式在加深学生对重要观点、原理的理解, 调动学生积极性, 提高学生解决 问题的能力等方面, 都具有明显的优势。《农业经济学》作为一门研究农业生产活动中生产力和生产关系 的运动规律的学科, 具有很强的应用性。而对中国这样一个农业大国的来说, 农业经济在中国经济占据着 举足轻重的地位, 为了合理解决目前农业经济发展中存在的各种问题, 就需要做好《农业经济学》课程的 教学工作, 培养更多的农业经济人才来服务于社会主义农业事业的发展。因此, 在《农业经济学》课程教 学中积极采用案例式教学模式, 具有重要的理论意义和实践意义。

教学评价是教学过程的最后环节, 其宗旨在于为前几个环节的活动提供信息反馈, 教学评价的有效开 展, 能帮助教师不断获得关于教学质量的动态信息, 及时获知教学活动的成败与教学中的主要问题, 适时 做出合理的调整, 从而不断提升自己的业务水准与教学效果。对于学生而言, 教学评价的有效开展, 能帮 
助学生客观地理解自己的学习效果并发现问题所在, 及时调整努力方向 [1]。目前, 已有许多教育工作者 就如何在《农业经济学》教学过程中开展案例教学模式进行了相关研究 [2-4]。但对于其教学效果的评价 多数属定性研究, 且实证分析不足, 缺乏详细和具体的讨论, 需要进一步的完善。另外, 教学评价是一个 多因素、多变量、模糊的非线性过程, 本文结合案例教学这一特点, 尝试将案例教学过程看成是一个齐次 马尔科夫链, 利用齐次马尔科夫链的极限分布, 排除了学习基础的差异, 从而实现对教师的在教学过程中 教学效果客观公正的评价。

\section{2 评价原理和方法}

利用马尔科夫链存在一个只与当前状态有关, 即只由变化原因确定的极限状态这个特点。本文把学生 的学习成绩分为若干种有限状态, 把整个教学过程分为若干个时间段, 则由上一个时间段到下一个时间段 的学生学习状态的变化, 就可以反映出教师的教学活动产生的教学效果, 这种效果是由学生现在所处的状 态向下一时间段状态的转化, 与其以前的状态无关, 因此整个教学过程就可以看成一个齐次马尔科夫链, 利用马尔科夫链的极限分布, 就可以对教师在这教学过程中的教学效果进行评价, 也可以对若干名教师的 教学效果进行比较 [5]。

\section{1 数据整理与学生成绩分级}

使用标准化考试试卷, 按照由低到高的顺序对学生成绩进行定级, 由于学习成绩低于 60 分的较少, 所以本研究将学生成绩划分为五个等级, 分别为 $0 \sim 59,60 \sim 69,70 \sim 79,80 \sim 89,90 \sim 100$ 分, 并计算 各等级学生人数占总人数的比例 (状态向量 $\mathrm{A}$ ), 其计算公示为 $A=\left(\frac{n_{1}}{n}, \frac{n_{2}}{n} \ldots . . \frac{n_{i}}{n}\right)$, 其中 ni 为第 $\mathrm{i}$ 等级 的学生人数。

\section{2 一步转移概率矩阵的计算}

根据马尔科夫的无后效性可知, 系统的下个状态只与当前状态有关, 而与更早之前的状态无关, 这时 转移概率矩阵 $\mathrm{P}$ 就成为一个重要的决定因子。在进行教学评价时, 转移概率矩阵 $\mathrm{P}$ 是教学质量等多种因素 的集中反映 $[6]$ 。由转移概率 $P_{i j}=\frac{n_{i j}}{n_{i}}$, 其中 $n i \mathrm{j}$ 表示学生成绩由 $\mathrm{i}$ 等级转到 $\mathrm{j}$ 等级的人数, 由于本文将学 生成绩划分为五个等级, 所以本文使用的一步转移概率矩阵 P 的计算公式为:

$$
P=\left(p_{i j}\right)_{5 \times 5}=\left(\frac{n_{i j}}{n_{i}}\right)_{5 \times 5}
$$

\section{3 教学效果评定}

长期以来、教学效果的评定是教学研究过程中的难点, 评定方法的选择决定着教学效果评定的准确性, 本文利用马尔科夫链的极限分布和罚因子法两个方法分别评定案例式教学模式在农业经济学教学中的教 师教学效果和教学质量。原因在于马氏链具有极限分布即非周期正常返不可约马氏链存在着唯一的平稳分 布 [7], 在教学水平等条件相当的情况下, 学生可能达到的各个级别的比例将会趋于稳定, 这种稳定只与 教学的水平有关, 与学生的基础无关, 符合教学效果评价的要求, 因此可以使用马尔科夫链的极限分布进 行教师教学效果的评定; 另外教学是教师和学生互动的过程, 仅从教师的教学效果来评价案例法的应用效 果又过于片面, 需要对学生在学习过程中成绩的变化情况进行分析, 以验证教学效果的优劣, 因此本文引 
入罚因子指标考察学生的成绩变化情况。

2.3.1 基于极限分布的教师教学效果评价

首先根据平稳方程 $\Pi P=\prod_{\text {且 }} \sum_{j=1}^{s} \pi_{j}=1$, 解出极限 $\Pi=\left(\pi_{1}, \pi_{2}, \pi_{3}, \pi_{4}, \pi_{5}\right)^{T}$, 最终教师的教学效果 指标方程为 $Y=\sum_{j=1}^{s} \pi_{j} x_{j}$, 其中 $\mathrm{xj}$ 指学生成绩。

2.3.2 基于罚因子的教学质量评价

首先将转移矩阵中的每个因子都乘以罚因子 $2(\mathrm{j}-\mathrm{i})$, 得出进步效率矩阵 $P^{*}=\left(2(j-i) \frac{n_{i j}}{n_{i}}\right)_{5 \times 5}$, 而 $E=\sum_{i=1}^{5} \sum_{j=l}^{5}\left(2(j-i) \frac{n_{i j}}{n_{i}}\right)_{\text {是学生成绩变化程度的累加, } \mathrm{E} \text { 越大, 表明教学质量就越好。 }}$

\section{3 案例式教学模式在《农业经济学》教学中的效果评价分析}

现有 2012 级农林经济管理专业和农村区域发展专业的学生（即甲班和乙班）各 30 人的两次（即期中 和期末）农业经济学的考试成绩。第一次成绩各等级人数统计（见如表 1 )。

表 1 第一次成绩及人数统计

\begin{tabular}{crrrrr}
\hline 人数/分数等级 & $0-59$ & $60-69$ & $70-79$ & $80-89$ & $90-100$ \\
\hline 甲班 & 0 & 2 & 10 & 15 & 3 \\
乙班 & 1 & 3 & 10 & 12 & 4
\end{tabular}

由第一次成绩及人数统计得初始状态向量:

$A_{\text {甲 }}\left(\begin{array}{lllll}0 & \frac{1}{15} & \frac{1}{3} & \frac{1}{2} & \frac{1}{10}\end{array}\right), A_{乙}\left(\begin{array}{llllll}\frac{1}{30} & \frac{1}{10} & \frac{1}{3} & \frac{2}{5} & \frac{2}{15}\end{array}\right) 。$

第一次考试后, 对乙班采用案例式教学模式开展教学活动, 而甲班则继续采用常规的教学方法, 经过 后半期的教学活动后, 第二次考试各等级人数转移情况见如表 2 和表 3 。

表 2 甲班第二次成绩及人数统计

\begin{tabular}{ccccccc}
\hline \multicolumn{7}{c}{ 第二次考试成绩 } \\
\cline { 3 - 7 } & & $90-100$ & $80-89$ & $70-79$ & $60-69$ & $0-59$ \\
\hline 第 & $90-100$ & 2 & 1 & 0 & 0 & 0 \\
一 & $80-89$ & 1 & 6 & 3 & 2 & 3 \\
次 & $70-79$ & 2 & 1 & 4 & 2 & 1 \\
考 & $60-69$ & 0 & 1 & 0 & 1 & 0 \\
试 & $0-59$ & 0 & 0 & 0 & 0 & 0 \\
成 & & & & & & \\
绩 & & & & & & \\
\hline
\end{tabular}


表 3 乙班第二次成绩及人数统计

\begin{tabular}{cccrrrr}
\hline & \multicolumn{2}{c}{ 第二次考试成绩 } & & & \\
\cline { 3 - 6 } & & $90-100$ & $80-89$ & $70-79$ & $60-69$ & $0-59$ \\
\hline 第 & $90-100$ & 3 & 1 & 0 & 0 & 0 \\
一 & $80-89$ & 3 & 5 & 3 & 1 & 0 \\
次 & $70-79$ & 0 & 4 & 3 & 3 & 0 \\
考 & $60-69$ & 0 & 0 & 1 & 1 & 0 \\
试 & $0-59$ & 0 & 0 & 1 & & 0 \\
成 & & & & & & \\
绩 & & & & & & \\
\hline
\end{tabular}

甲班和乙班学生成绩的一步转移矩阵分别为:

$$
P_{\text {甲 }}=\left(\begin{array}{ccccc}
\frac{2}{3} & \frac{1}{3} & 0 & 0 & 0 \\
\frac{1}{15} & \frac{2}{5} & \frac{1}{5} & \frac{2}{15} & \frac{1}{5} \\
\frac{1}{5} & \frac{1}{10} & \frac{2}{5} & \frac{1}{5} & \frac{1}{10} \\
0 & \frac{1}{2} & 0 & \frac{1}{2} & 0 \\
0 & 0 & 0 & 0 & 0
\end{array}\right), \quad P_{乙}=\left(\begin{array}{ccccc}
\frac{3}{4} & \frac{1}{4} & 0 & 0 & 0 \\
\frac{1}{4} & \frac{5}{12} & \frac{1}{4} & \frac{1}{12} & 0 \\
0 & \frac{2}{5} & \frac{3}{10} & \frac{3}{10} & 0 \\
0 & 0 & \frac{1}{3} & \frac{1}{3} & \frac{1}{3} \\
0 & 0 & 1 & 0 & 0
\end{array}\right)
$$

由转移矩阵 P 甲得甲班学生成绩的平稳方程组:

$$
\Pi=\left(\pi_{1}, \pi_{2}, \ldots, \pi_{5}\right)=\left(\pi_{1}, \pi_{2}, \ldots, \pi_{5}\right) P_{\text {甲 }}=\left(\begin{array}{ccccc}
\frac{2}{3} & \frac{1}{3} & 0 & 0 & 0 \\
\frac{1}{15} & \frac{2}{5} & \frac{1}{5} & \frac{2}{15} & \frac{1}{5} \\
\frac{1}{5} & \frac{1}{10} & \frac{2}{5} & \frac{1}{5} & \frac{1}{10} \\
0 & \frac{1}{2} & 0 & \frac{1}{2} & 0 \\
0 & 0 & 0 & 0 & 0
\end{array}\right), \pi_{j}>0, \sum_{j=1}^{s} \pi_{j}=1
$$

由转移矩阵 P 乙得乙班学生成绩的平稳方程组:

$$
\Pi=\left(\pi_{1}, \pi_{2}, \ldots, \pi_{5}\right)=\left(\pi_{1}, \pi_{2}, \ldots, \pi_{5}\right) P_{乙}=\left(\begin{array}{cccccc}
\frac{3}{4} & \frac{1}{4} & 0 & 0 & 0 \\
\frac{1}{4} & \frac{5}{12} & \frac{1}{4} & \frac{1}{12} & 0 \\
0 & \frac{2}{5} & \frac{3}{10} & \frac{3}{10} & 0 \\
0 & 0 & \frac{1}{3} & \frac{1}{3} & \frac{1}{3} \\
0 & 0 & 1 & 0 & 0
\end{array}\right), \pi_{j}>0, \sum_{j=1}^{s} \pi_{j}=1
$$

分别解甲班和乙班学生成绩两平稳方程组得极限向量 


$$
\begin{aligned}
& \prod_{\text {甲 }}=\left(\begin{array}{lllll}
0.169 & 0.423 & 0.141 & 0.169 & 0.098
\end{array}\right), \\
& \prod_{乙}=\left(\begin{array}{lllll}
0.390 & 0.390 & 0.173 & 0.035 & 0.012
\end{array}\right)
\end{aligned}
$$

最后赋予 5 个等级学分绩点分别为 $95 、 84.5 、 74.5 、 64.5 、 29.5$, 令 $Y_{j}=\pi{ }_{j}\left(x_{1}, x_{2}, x_{3}, x_{4}, x_{5}\right)^{T}$ 为班 级 $j$ ( $j=$ 甲或乙) 所对应教师的综合评估值, 得到 $Y$ 甲 $=76.0945, Y$ 乙 $=85.505$ 。由于 $Y$ 甲 $<Y$ 乙, 认为乙 班教师的教学效果优于甲班的, 这与乙班通过运用了案例式教学模式, 使教学效果得到改善的实际相一致。 将每个因子 pij 都乘以罚因子 $2(j-i)$, 得出新的转移矩阵分别为:

$$
\begin{gathered}
S_{\text {甲 }}=\left(\begin{array}{ccccc}
0 & \frac{2}{3} & 0 & 0 & 0 \\
-\frac{2}{15} & 0 & \frac{2}{5} & \frac{8}{15} & \frac{6}{5} \\
-\frac{4}{5} & -\frac{1}{5} & 0 & \frac{2}{5} & \frac{2}{5} \\
0 & -2 & 0 & 0 & 0 \\
0 & 0 & 0 & 0 & 0
\end{array}\right), S_{乙}=\left(\begin{array}{ccccc}
0 & \frac{1}{2} & 0 & 0 & 0 \\
-\frac{1}{2} & 0 & \frac{1}{2} & \frac{1}{3} & 0 \\
0 & -\frac{4}{5} & 0 & \frac{3}{5} & 0 \\
0 & 0 & -\frac{2}{3} & 0 & \frac{2}{3} \\
0 & 0 & -4 & 0 & 0
\end{array}\right) 。 \\
\text { 通过计算得出两个班级的效率度分别为 } E_{\text {甲 }}=\frac{7}{15}, E_{乙}=\frac{91}{30} \text { 。 }
\end{gathered}
$$

由于 $\mathrm{E}$ 甲 $<\mathrm{E}$ 乙, 认为乙班教学的效率度优于甲班的, 即乙班考试成绩进步的学生数量多于甲班的, 而甲班教学的效率度数值较少, 表明采用案例式教学模式的乙班学生的成绩进步的学生数量多, 未采用案 例式教学模式的甲班学生的成绩等级转化不够显著。

\section{4 结论与启示}

《农业经济学》作为一门实践性和应用性都较强的专业学科, 如果在课堂上强行灌输相关理论, 缺乏 实际的案例讲解, 一方面学生学习必将陷入死记硬背的窠臼, 同时根本无法学习到农业经济学中的思维方 式和先进的理念, 教学效果必然不好, 而案例教学法在培养学生独立思考能力、全面分析能力以及创新能 力等方面有着突出的优势, 具有较好的应用前景。从本文对案例式教学模式在《农业经济学》教学中的应 用效果来看, 采用了案例式教学模式的乙班不管是从教师的效果指标还是学生的进步指标上都好于甲班, 表明案例式教学模式在《农业经济学》课程教学中有较好的效果, 应该大力推广。

在教学评价过程中, 评价标准的选择往往较为困难, 通常的做法是以学生考试的及格率和平均成绩来 评价教学效果, 这种方法没有考虑学生原始成绩对教学效果的影响, 这显然有较大的局限性。由于马尔科 夫具有无后效性, 通过引入马尔科夫链对采用案例式教学模式之后学生成绩的相对提高展开教学效果的评 价, 能够真正反应教师的教学效果和学生的进步水平。本文分析了案例式教学模式在《农业经济学》课程 教学中的应用效果, 通过极限分布和罚因子法评价教师的教学效果, 两种方法互相印证了案例式教学模式 对学生在学习《农业经济学》课程过程中成绩提高的促进作用, 这种以考虑学生成绩和教学效果之间的关 系为目的的评价体系, 比以往将考试分数的平均分、合格率等为标准进行教学效果评估的模式, 更具有较 高的科学性和实用性 [6], 表明基于马尔科夫链评价教师的教学效果具有很好的可操作性, 可以广泛用于 各学科的教学评价。 


\section{References}

[1] Y.Q.Ji: Curriculum and Teaching Theory[M], Nanjing University Press, 2009:170-171.(In Chinese)

[2] W.C.Geng: Application Analysis of Case Teaching Method in Agricultural Economics[J], China Collective Economy, 2009,30:187-188.(In Chinese)

[3] L.L.Gu and H.M.Jiang: Application Analysis of Case Teaching in Agricultural Economics[J], Modern Communication, 2012,02:252-253.(In Chinese)

[4] Y.K.Zhao and C.P.Zhao: The Application and Effect Analysis of Participatory Teaching Method in the Teaching of Agricultural Economics[J], Journal of Henan Institute of Science and Technology, 2013,12:95-97.(In Chinese)

[5] H.Zhang: An application of Markov chain[J], Journal of Changchun Institute of Optics and Fine Mechanics, 1994,03:44-49.(In Chinese)

[6] L.W.Liu, X.R.Chen and T.He: Teaching Effect Evaluation Method Based on Markov Chain[J], Statistics and Decision, 2014,03:93-94.(In Chinese)

[7] L.Y.Wan: The Application of Markov Chain in the Evaluation of Teaching Quality[J]. Journal of Nantong Vocational University, 2011,02:67-69.(In Chinese) 